\title{
Oral tranexamic acid in the treatment of melasma: A prospective study in a Moroccan population
}

\author{
Fouzia Hali, Fatima-Zahra Kettani, Kenza Baline, Soumia Chiheb
}

Department of Venereology and Dermatology, CHU Ibn Rochd, Casablanca, Morocco

Corresponding author: Dr. Fatima-Zahra Kettani, E-mail: kettani.fatimazohra@gmail.com

\begin{abstract}
Background: Melasma is a common pigmentary disorder. Its treatment is a real challenge. Objective: The aim of the study was to evaluate the effectiveness of oral tranexamic acid (TA) in melasma in a Moroccan population. Methods: Prospective study in the dermatology department of Ibn Rochd University Hospital of Casablanca from January 2018 to February 2019. The oral TA was administered at a dose of $250 \mathrm{mg}$ twice a day with a photo external protection. The percentage reduction in the initial value of the Melasma Area and Severity Index (MASI) was evaluated every 4 weeks. Photographs were taken and analyzed using the Visioface device. Results: 17 women were enrolled. The average age was 40 years old with extremes ranging from 27 to 53 years old. All patients were skin phototype 3 or 4. The average duration of treatment was 6 months. Sunlight exposure was found in 10 patients, oral contraception in 7 patients, three patients had an intrauterine device, family history of melasma was found in 6 patients. The evaluation at 6 months showed a good response in 10 patients (66,7\%), moderate response in 3 patients (20\%) and slight response in 2 patients (13,3\%). VisioFace analysis showed an overall improvement in all patients. The treatment was well tolerated with no adverse effects. Conclusion: Our study, although limited by the small number of samples, proves that oral AT may be a promising therapeutic option. Larger studies with longer treatment durations as well as studies comparing TA with other treatments are needed.
\end{abstract}

Key words: Melasma; Oral tranexamic acid; Hyperpigmentation

\section{INTRODUCTION}

Melasma is a benign pigmentary disorder that is especially common in Moroccan women. The pathogenesis of melasma is still unclear and its treatment remains a challenge. Existing treatment modalities include prevention of UV radiation, topical lightening agents, chemical peel, and light-based and laser therapies. However, incomplete clearance and frequent recurrences make melasma a frustrating condition [1].

Recently, tranexamic acid (TA) has been reported to be effective for melasma in several studies.

TA is an anti-fibrinolytic agent that inhibits plasminogen activation through the reversible blockade of lysinebinding sites on plasminogen molecules. The inhibition of this plasminogen-plasmin system inhibits melanin synthesis by interfering with melanocyte-keratinocyte interactions, and forms the biological basis of the skinlightening properties of TA [2].

The aim of this study was to evaluate the therapeutic effects of oral TA in the treatment of melasma in a Moroccan population.

\section{MATERIALS AND METHODS}

We conducted a prospective study in the dermatology department of Ibn Rochd University Hospital of Casablanca from January 2018 to February 2019.

The criteria for inclusion selected female patients with melasma.

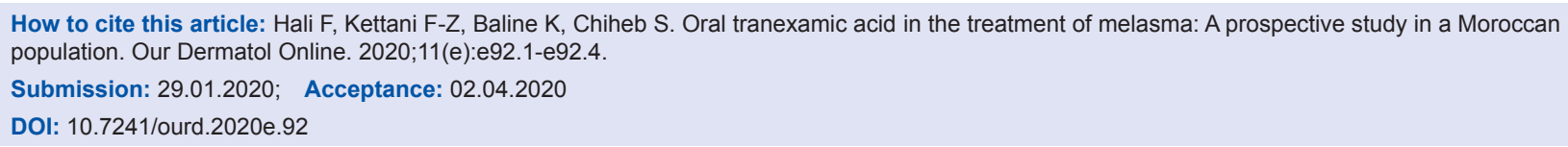


The exclusion criteria ruled out women with a pregnancy or a breastfeeding period, a history of severe renal failure, thrombophilic disorders or thromboembolic disease, or hypersensitivity to TA.

A total of 17 patients with melasma were enrolled. The oral TA was administered at a dose of $250 \mathrm{mg}$ twice daily for a period of 6 months. All patients received sunscreen SPF 50 with instructions to apply every morning, with reapplication every 2 hours during daylight hours, without any additional medicine for the treatment of melasma.

A detailed questionnaire recorded the comorbid medical history, current medical history, sunlight exposure, related medicine administrations, former treatments, cosmetics usage and related habits. Our patients were informed about the risks, benefits, and potential complications.

Photographs were taken and analyzed using the VisioFace ${ }^{\circledR}$ device before the initiation of the treatment, at 3 months and at 6 months.

The percentage reduction in the initial value of the Melasma Area and Severity Index (MASI) was evaluated every 4 weeks, and the response was considered very good ( $>75 \%$ reduction), good $(50 \%$ $<75 \%$ reduction)), moderate ( $25 \%$ at $<50 \%$ reduction), slight ( $<25 \%$ reduction) or without response.

At the end of the study, we collected the degree of satisfaction of the patients.

\section{Ethics Statement}

All patients were consentants

\section{RESULTS}

Of 17 patients, 15 completed the trail. The average age was 40 years old with extremes ranging from 27 to 53 years old. All patients were Fitzpatrick skin types III or IV.

The average duration of melasma was 7.9 years (range: 2 to 20 years).

The risk factors found were sunlight exposure (10 patients), oral contraception (7 patients), and family history of melasma (6 patients). Three patients had an intrauterine device and 4 patients reported that melasma appeared during pregnancy.
Regarding previous treatments for melasma, 13 patients $(86,6 \%)$ were treated with depigmenting creams, 3 patients $(20 \%)$ with chemical peels and 1 patient $(6,6 \%)$ with laser therapy. Two patients $(13,3 \%)$ reported no previous treatment for melasma.

The evaluation at 3 months showed good response in 8 patients $(53,4 \%)$, a moderate response in 5 patients $(33,3 \%)$ and slight response in 2 patients $(13,3 \%)$.

After 6 months of treatment, the evaluation showed good response in 10 patients $(66,7 \%)$, moderate response in 3 patients $(20 \%)$ and slight response in 2 patients $(13,3 \%)$

Thus, the total improvement rate for melasma was 86,7 $\%$ of the subjects.

No deterioration of melasma was found during the treatment.

Visioface analysis showed an overall improvement in all patients.

Representative clinical photographs are included in (Figs. la and lb).

Significant improvements of the quality of life were observed in patients' self-assessment.

The treatment was well tolerated and no side-effects were reported during the treatment and throughout the follow-up period.

However, $40 \%$ of the patients had a relapse of melasma within 3 months of ceasing TA.

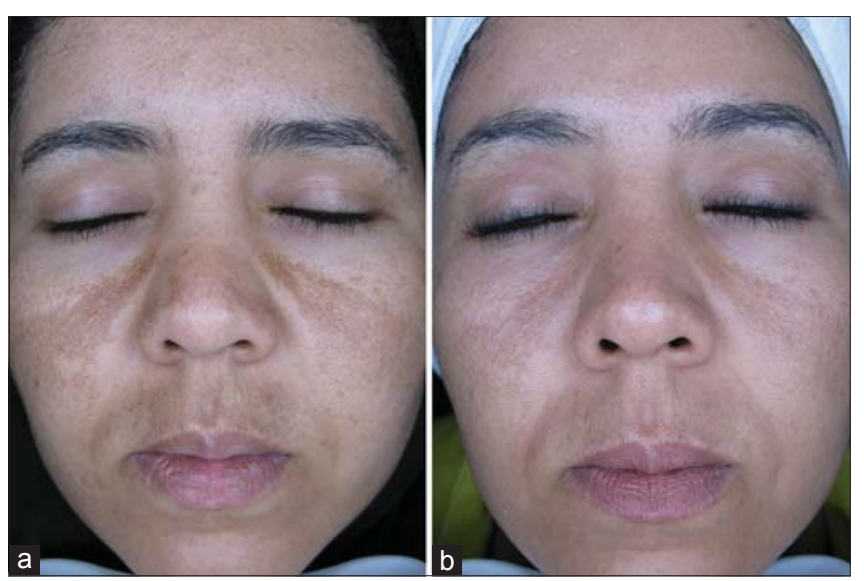

Figure 1: Clinical photographs of a melasma patient at the first visit, (a) before initiation of treatment and (b) after 6 months of treatment. 


\section{DISCUSSION}

Melasma can be seen frequently among Moroccan women. Its rate of incidence was not clear in Morocco but was about $3 \%$ in the United States [3].

First-line treatment usually consists of topical compounds that interfere with the melanin synthesis, broadspectrum photoprotection, and camouflage. Chemical peels are often added in second-line therapy. Laser and light therapies represent potentially promising options for patients who are refractory to other modalities, but also carry a significant risk of worsening the disease [4].

Although TA therapy is becoming a usual method for the treatment of melasma in Asia [3], we did not find any studies on a Maghrebi population, in this case Moroccan.

The most common therapy of melasma had been the external use of hydroquinone cream. However, the use of hydroquinone is associated with a higher frequency of adverse effects including burning, itching, dryness, erythema, contact dermatitis, and more importantly, toxic reactions such as permanent "confetti-like" depigmentation. For these reasons, hydroquinone has already been banned in Japan, the European Union, Australia, and China [3]. In our Moroccan context, patients often use topical herbs and creams sold in illegal markets with an unclear composition often leading to these same undesirable effects with even more severe post inflammatory hyperpigmentations.

The exact pathogenesis of melasma remains unknown. Studies have implicated several factors that cause melanocytes to become activated and produce excessive melanin in patients with melasma. These factors include sun exposure, hormones, genetic influences, and vascular influences [2-5]. Histologically, lesional skin has increased melanocytes, melanosomes, solar elastosis, mast cells, blood vessels, vascular endothelial growth factor (VEGF), 3 c-kit, and kit ligand. A disrupted and thinner basement has also been reported [5].

In this study, the most common reasons for melasma pathogenesis were sunlight exposure, pregnancy, heredity, and contraceptives. It is important to let patients know that the exclusion of these factors may be helpful to the improvement of melasma.
Although epidermal hyperpigmentation is the key feature in melasma, increasing evidence supports the role of an epidermal barrier dysfunction, keratinocytemelanocyte interactions and dermal factors in this condition. Increased vascularity, which is not adequately addressed with conventional topical lightening treatments, is another factor that has been consistently found in lesional melasma skin [2].

Tranexamic acid, an antifibrinolytic agent, has emerged as a promising treatment for melasma. TA was originally developed for the treatment of menorrhagia and bleeding diathesis. It was found that patients on TA for these indications in Asia had improvement of melasma. Subsequently, it was found that TA causes decreased tyrosinase activity in melanocytes, possibly due to decreased production of fibroblast growth factor (FGF) and prostaglandins by blocking the conversion of plasminogen to plasmin. Both FGF and prostaglandins are potent stimulants of melanocyte activity [5].

TA has also been shown to reduce UV radiationinduced pigmentation by decreasing UV-induced plasmin activity and subsequent arachidonic acid release and to decrease mast cell activity. Furthermore, TA can inhibit neovascularisation induced by basic fibroblast growth factor, as plasmin is important in angiogenesis by converting extracellular matrix-bound vascular endothelial growth factor into freely diffusible forms [2].

When TA is used as a hemostatic, it is prescribed at a dosage of 1,000 $\mathrm{mg}$ three times daily, whereas in the treatment of melasma, it is used at the dosage of $250 \mathrm{mg}$ twice daily, which is only one sixth of the normal dosage. At this dose, several adverse effects have been reported in various studies such as gastrointestinal irritations (nausea, diarrhea, and abdominal pain), hypomenorrhea, headaches, and back pain $[3,5]$.

The current study supports the efficacy of oral TA, taken at a low dose of $250 \mathrm{mg}$ twice daily, in lightening melasma. Our patients achieved $86,7 \%$ lightening of MASI scores with no significant complications.

Melasma is known to have high relapse rates [6]. In our study, $40 \%$ of the patients had a relapse of melasma within 3 months of ceasing TA, despite continuing photoprotection. Tan et al prosposed that treatment should be confined to a defined duration and instituted in conjunction with judicious photoprotection triple 
combination topical therapy, in order to minimise relapses after discontinuation [2].

\section{Limitations of Study}

We conducted a prospective study without a control group because it is difficult to enroll a significant number of volunteers to take the TA tablets during a period of 6 months especially since our study was not funded.

\section{CONCLUSION}

In conclusion, this study indicates that oral TA acid seems to be a promising and safe therapeutic option. But large-scale and multicenter trials are required to evaluate the longterm administration and the effect of combining this drug with other treatments to identify any additive effect in the search for better treatments for melasma.

\section{Statement of Human and Animal Rights}

All procedures followed were in accordance with the ethical standards of the responsible committee on human experimentation (institutional and national) and with the Helsinki Declaration of 1975 , as revised in 2008 .

\section{Statement of Informed Consent}

Informed consent was obtained from all patients for being included in the study.

\section{REFERENCES}

1. Zhou LL, Baibergenova A. Melasma: systematic review of the systemic treatments. Int J Dermatol. 2017;56:902-8.

2. Tan AWM, Sen P, Chua SH, Goh BK. Oral tranexamic acid lightens refractory melasma. Australas J Dermatol. 2017;58:e105-8.

3. Wu S, Shi H, Wu H, Yan S, Guo J, Sun Y, et al. Treatment of melasma with oral administration of tranexamic acid. Aesthetic Plast Surg. 2012;36:964-70.

4. Taraz M, Niknam S, Ehsani AH. Tranexamic acid in treatment of melasma: A comprehensive review of clinical studies: TARAZ et al. Dermatol Ther. 2017;30:e12465.

5. Del Rosario E, Florez-Pollack S, Zapata L, Hernandez K, TovarGarza A, Rodrigues M, et al. Randomized, placebo-controlled, double-blind study of oral tranexamic acid in the treatment of moderate-to-severe melasma. J Am Acad Dermatol. 2018;78:363-9.

6. Lee HC, Thng TGS, Goh CL. Oral tranexamic acid (TA) in the treatment of melasma: A retrospective analysis. J Am Acad Dermatol. 2016;75:385-92.

Copyright by Fouzia Hali, et al. This is an open access article distributed under the terms of the Creative Commons Attribution License, which permits unrestricted use, distribution, and reproduction in any medium, provided the original author and source are credited.

Source of Support: Nil, Conflict of Interest: None declared. 